\title{
Quantitative trait loci (QTLs) linked with root growth in lettuce (Lactuca sativa) seedlings
}

by Roberts, J., Broadley, M.R., Pink, D., Hand, P., Lynn, J. and Monaghan, J.M.

Copyright, publisher and additional information: .This is the authors' accepted manuscript. The published version is available via Springer Link

Please refer to any applicable terms of use of the publisher.

DOI: https://doi.org/10.1007/s11032-019-1089-x 


\section{Quantitative trait loci (QTLs) linked with root growth in lettuce (Lactuca} sativa) seedlings.

J. Roberts ${ }^{1}$, M.R. Broadley ${ }^{2}$, D. Pink ${ }^{1}$, P. Hand ${ }^{1}$, J. Lynn ${ }^{3}$ \& J.M. Monaghan ${ }^{1 *}$

${ }^{1}$ Fresh Produce Research Centre, Crop and Environment Department, Harper Adams University, UK.

${ }^{2}$ Plant and Crop Sciences Division, School of Biosciences, University of Nottingham, UK.

${ }^{3}$ Applied Statistical Solutions Ltd, Leamington Spa, UK.

*Corresponding author (jmmonaghan@harper-adams.ac.uk).

\section{Abstract}

In-field variation of transplanted lettuce (Lactuca sativa L.) due to variable soil and environmental conditions is one of the major restrictions in the optimization of production and yield. Marker assisted breeding for lettuce varieties with a more rapid rooting phenotype has the potential to improve the performance of lettuce transplants. This study aimed to identify traits linked with increased primary root length, lateral root length and lateral root emergence in $14 \mathrm{~d}$ L. sativa seedlings from an intra-specific cross (Saladin $\mathrm{x}$ 
Iceberg). In total 16 significant quantitative trait loci (QTLs) were associated with increased root growth traits that would allow direct introgression of the traits. Six of the QTLs were associated with increased primary root growth, accounting for $60.2 \%$ of the genetic variation for the trait. Three QTLs were associated with lateral root growth (38.6 \% of genetic variation); two QTLs were associated with lateral root length density (27.6\% of genetic variation) and three with root number density (33.4 \% of genetic variation) and two QTLs were associated with mean lateral root length ( $21.1 \%$ of genetic variation). The statistical QTLs were located across 9 different linkage groups (LGs) representing loci on 7 of the 9 L. sativa chromosomes. A combination of restriction fragment length polymorphism (RFLPs) and Kompetitive allele specific PCR (KASPs) markers linked to these rooting traits were identified, which could allow breeders to select for a rapid establishment phenotype. population, Transplant, Root traits, Quantitative trait loci.

Key words: Lactuca sativa, Rapid rooting, Establishment, Mapping Author contribution statement J.R; Carried out the main body of the research and main author of the paper. J.M; Principle research and paper advisory.

M.R.B; Secondary research and paper advisory. D.P; Secondary research and paper advisory. P.H; principle advisory for QTL analysis and secondary paper advisory. 
J.L; Principle advisory for the statistical analysis of trait data.

\section{Key message}

The study has identified genotypic variation for root growth traits within cultivated lettuce that will allow direct introgression of these traits into commercial cultivars for improved uniformity and establishment.

\section{Acknowledgements}

The research undertaken in this study was conducted primarily at Harper Adams University. We would like to thank our collaborative funding bodies; Syngenta, Harper Adams University and the University of Nottingham and the Vegetable Genetic Improvement Network for providing the plant materials to use in this study. A special thank you goes to Catherine Thomas who helped with the initial modification of the high-throughput platform for the use with lettuce seedlings, Rory Hayden for delivery of the equipment to Harper Adams University and Lolita Wilson for all her support at The University of Nottingham. Gratitude also goes to Shelley Roberts for her help with placement of the seedlings within the platform.

\section{Introduction.}

In Europe and North America, lettuce (Lactuca sativa L.) seedlings are typically grown during the early stages of production in glasshouses prior to transplanting out into the field. This removes issues associated with direct drilled seed such as, germination, crop uniformity and avoidance of early weed infestation, while optimizing growth and yield (Sharma et al. 2005; Maltais et al. 2008).

Transplant establishment requires the regeneration of new roots and resumption of shoot growth in the field following transplanting (Orzolek 1991). Transplanted crops 
differ morphologically from direct drilled crops with loss of the tap root resulting in the development of a larger number of lateral roots (NeSmith \& Duvall 1998).

Each lettuce plant within a crop needs to achieve similar establishment to give as uniform a crop as possible for the optimization of production. Lettuce is still manually harvested and growers will only carry out 'once-over' harvest therefore crop uniformity is essential for profit. Transplant establishment can be negatively impacted by many factors within a field. For example, the variability of soil parameters, such as $\mathrm{pH}$ can reduce nutrient availability and root growth (Orzolez 1991). Compaction and poorly tilled soil result in poor root penetration (Grassbaugh \& Bennett 1998). Soil moisture can be too high or low for adequate root development (Grassbaugh \& Bennett 1998). Transplant shock, which describes the sudden transient stresses at transplanting (Kerbiriou et al. 2013), such as temperature change can also impact establishment. Better establishment would improve crop uniformity by minimising the variation between plants caused by abiotic stress at the time of transplanting through the rapid establishment of young plants and the associated access to nutrient and water (Johnson et al. 2000).

As for most crops, lettuce breeding has to date been focused on yield, leaf/head traits and pest and disease resistance with little or no direct attention given to the root system. A root breeding strategy in lettuce would be to identify quantitative trait loci (QTLs) linked to beneficial root growth traits and introduce these into crop varieties through marker assisted selection breeding programmes to develop lettuce cultivars capable of rapid establishment under variable soil conditions. The introduction of root trait QTLs has been previously shown to be successful in upland rice (Oryza sativa), where root traits for longer and broader roots were introduced into a new variety which improved yields (Steele et al. 2006; 
95 Steele et al. 2013). Identifying genetic resources that allow lettuce cultivars to

96 achieve uniform establishment will be of great importance as future more

97 'sustainable' crop production will most likely be carried out under conditions of lower

98 fertilizer and water use (Zhu et al. 2011) and increased fertilizer prices as nutrients

99 such as phosphorus diminish (Le Marié et al. 2014).

100 Previously, QTLs based on segregating root traits have been identified in two 101 studies on lettuce. Both studies used an inter-specific cross between cv. Salinas and 102 the wild relative Lactuca serriola (Johnson et al. 2000; Wei et al. 2014). The first 103 study analysed drought tolerance through deep soil water exploitation and identified 104 QTLs involved with root growth and biomass (Johnson et al. 2000). The second 105 study analysed salt tolerance in seedlings through changes to root system 106 architecture (Wei et al., 2014). Both studies demonstrated that a number of Lactuca 107 species root traits are under genetic control in seedling assays. However, it is not 108 known whether these traits are related to a rapid rooting phenotype. The study 109 reported here utilised a high-throughput growth pouch assay to analyse root growth 110 traits in an intra-specific cross mapping population with the aim of identifying QTLs 111 associated with an increased root growth phenotype in $14 \mathrm{~d}$ old seedlings that may 112 then be used for marker assisted breeding for the improvement of lettuce transplant 113 establishment. between the crisphead L. sativa cv Saladin (syn Salinas) bred in the US and the

118 Batavian L. sativa cv lceberg, bred in France (Atkinson et al. 2013). The mapping 
population used in this study for QTL analysis consists of $125 \mathrm{~F}_{8}$ recombinant inbred lines (RILs) that were selected as the most genetically informative subset from 254 $F_{5}$ genotyped individuals (Atkinson et al. 2013).

\section{Seed germination.}

Germination paper (SD7640; Anchor Paper Company, St Paul, MN, USA) was placed in petri dishes with 10 numbered sections marked out with a pen (Fig 1a). The germination papers were pre-soaked with $7 \mathrm{ml}$ of tap water for imbibition of the seed. Once the seeds had been placed on the sections they were placed in a $310 \times 340 \mathrm{~mm}$ lidded opaque plastic tray and held in a cold store $\left(14-16^{\circ} \mathrm{C}\right)$ with $24 \mathrm{~h}$ low irradiance lighting $\left(1.5 \mu \mathrm{mol} \mathrm{m} \mathrm{m}^{-2} \mathrm{~s}^{-1}\right.$ photosynthetically active radiation (PAR)). The seeds were left for up to $48 \mathrm{~h}$ to reach a pre-determined stage of germination, which was defined as the presence of a radicle $1-5 \mathrm{~mm}$ long and initial root hairs that formed an arrowhead-like appearance (Fig 1b). This assured all seedlings were placed on any given assay at the same growth stage, removing any variation due to germination time.

\section{High through-put growth pouch assay.}

A high through-put growth pouch assay (Atkinson et al. 2015; Thomas et al. 2016) was constructed as described by Thomas et al. (2016) but modified for use with lettuce by the inclusion of two sheets of porous tissue paper (TFM Farm and Country Superstore Ltd, Shropshire, UK), which increased water availability to the seedlings. Germinated seeds were placed at the top of the growth pouch with the radicle orientated towards the bottom of the paper (Fig 1c), with 2 seeds on each side of the pouch at approximately $15 \mathrm{~cm}$ spacing (Fig $1 \mathrm{~d}$ ). The growth pouches were suspended over drip trays supported within an aluminium frame as described 
by Atkinson et al. (2015). Each drip tray had $2 \mathrm{~L}$ of tap water containing $15 \%(0.24 \mathrm{~g}$

$\mathrm{L}^{-1}$ ) Hoagland's solution (Hoagland's No. 2 Basal Salt Mixture, Sigma Aldrich, Dorset UK) added. Above each tank were six $550 \mathrm{~mm}$ strip white light emitting diode (LED) lights (Leyton Lighting, Essex, UK) providing a mean PAR of $90.1 \mu \mathrm{mol} \mathrm{m} \mathrm{m}^{-2} \mathrm{~s}^{-1}$, ranging from 68.5-113.4 $\mu \mathrm{mol} \mathrm{m} \mathrm{m}^{-2} \mathrm{~s}^{-1}$.

\section{Seedling growth.}

Following germination six replicate growth pouches of each genotype were allocated to positions in the support frames using a one-way design with no blocking (GenStat $17^{\text {th }}$ edition, VSN International Ltd, Hemel Hempstead, UK). The seedlings were grown across two frames for a $14 \mathrm{~d}$ period with a $20 \mathrm{~h}$ photoperiod. The temperature and relative humidity $(\mathrm{RH})$ was recorded every 2 hours with a data logger (TinyTag Plus2, Gemini Data Loggers Ltd, Chichester, UK). The mean temperature was $13.8^{\circ} \mathrm{C}$ and ranged between $13.6^{\circ} \mathrm{C}$ and $18^{\circ} \mathrm{C}$. The mean $\mathrm{RH}$ was $99.2 \%$ with a minimum of $78.7 \%$ and a maximum of $100 \%$. Following $14 \mathrm{~d}$ growth the pouches were removed from the system for imaging.

\section{Image analysis.}

The growth pouches were removed from the frame and dismantled to expose the root system. The root system was then imaged with a digital camera (Lumix DMC-FP2, Panasonic, Berkshire, UK) at fixed distance of $200 \mathrm{~mm}$. The images were analysed using ImageJ (Abràmoff et al. 2004; Schneider et al. 2012) and measurements for primary root length, total lateral length and number of laterals were recorded and analysed. 


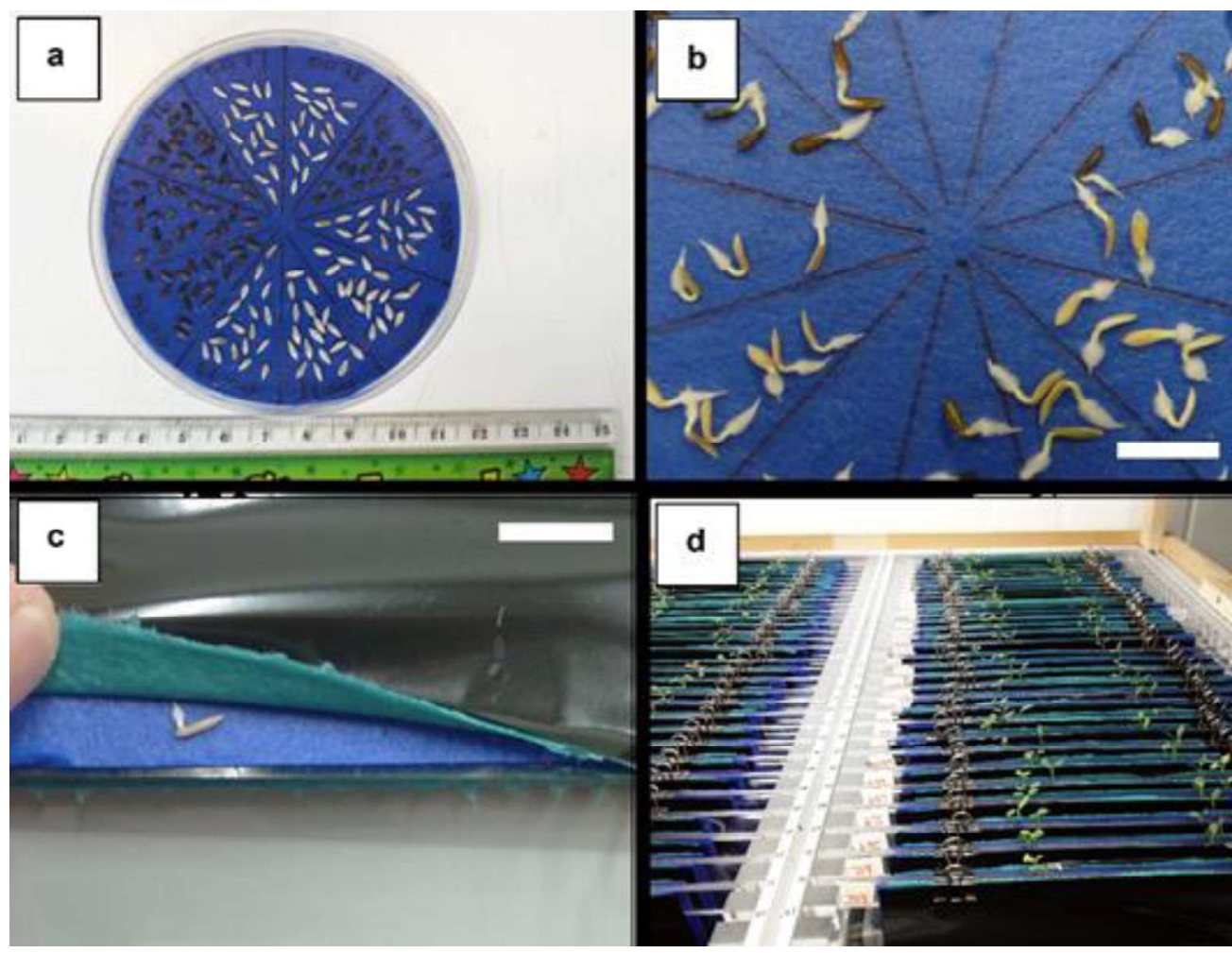

Fig. 1 Seed germination, position and growth in the pouch assay.

The imbibed seed on the germination paper in a petri dish (a). The

predetermined stage at which the germinated seedlings were placed in the

An increase in root growth resulted in an increase in the variance of the

residuals indicating that the data had non-constant variance and was not normally 
distributed. The raw data for the RILs and the parental lines were therefore transformed to square root and the mean calculated to normalise the distribution of the data for statistical analysis.

The transformed data were analysed using restricted maximum likelihood (REML) variance component analysis which accounted for variation, such as light level or edge effect that occurred within the frames. The resultant predicted means for all lines were then analysed to determine significant differences between genotypes. From the three measured phenotypes; primary root length (PRL), total lateral root length (TLL) and total number of lateral roots $(T N L)$ three further ratios were produced, which were lateral root length density $(L R L D=T L L / P R L)$, lateral root number density $(L R N D=T N L / P R L)$ and the mean lateral root length $(M L R L=$ $T L L / T N L)$. Broad sense heritability $\left(\mathrm{H}^{2}\right)$ for each trait was calculated from the variance component analysis $(V G /(V E+V R)$ where $V G$ is the genotypic variance, VE the sum of the component variance and VR is the residual variance). All statistical analysis of the mapping population data was done using GenStat 17th edition (VSN international Ltd, Hemel Hempstead, UK).

\section{QTL analysis.}

Restriction fragment length polymorphism (RFLP) and Kompetitive allele specific PCR (KASP) markers were used to genotype both the parents and the RIL population. The RFLP markers were previously published for the Saladin x Iceberg linkage map (Atkinson et al. 2013). Additional KASP markers were derived from single nucleotide polymorphisms (SNPs) between the genomic sequences of the parent accessions. To identify SNPs, Oligo(dT) selection of mRNA was performed twice from total RNA extracts from each parental line of the RIL population using 
202 Dynal magnetic beads (Invitrogen-ThermoFisher Scientific, Massachusetts, USA)

203 according to the manufacturer's instructions. Sequencing libraries were prepared

204 using mRNA-TruSeq sample prep kit v5 (Illumina Inc., San Diego, USA) according to

205 the manufacturer's protocol (15018818 revA). These libraries were sequenced using

206 Illumina's GAllx sequencing system. Using a CASAVA pipeline, 70 base paired-end

207 sequence reads were base-called and scored for read quality.

208 The linkage maps were constructed using JoinMap4 (Kyazma B.V,

209 Wageningen, The Netherlands). Following REML transformation of the data the

210 predicted mean values for all traits were analysed using MapQTL6 (Kyazma B.V,

211 Wageningen, The Netherlands). Initially the data were analysed using interval

212 mapping to identify putative QTLs (Zeng 1994) before further analysis was done

213 using multiple QTL model (MQM) mapping, adding significant cofactor markers to

214 eliminate genetic variation (background noise) caused by QTLs located elsewhere

215 on the genome (Jansen \& Stam 1994). The statistical logarithm of odds (LOD) score

216 was calculated for a genome wide and chromosome wide significance of $P<0.05$ (1 -

$217 \alpha c=\sqrt[n]{(1-\alpha g)}$, where $\alpha c$ is the chromosomal significance threshold, $\alpha g$ is the

218 genome wide significance threshold and $n$ is the number of chromosomes) (van

219 Ooijen 1999).

\section{Results.}

Some individual seedlings did not emerge, had severely inhibited primary root

growth or browning of the root tissue. These seedlings were not included in the data

223 analysis (99 seedlings from a total of 726 ). In total 42 lines had one data point

224 missing, 19 lines had 2 data points missing, 5 lines had 3 data points missing and 1

225 RIL (RIL 36) had 4 data points missing. 
227 mapping population, including the parents, for all six root traits; primary root length

$228(\mathrm{SEM}=0.041$, Fig 2a); total lateral root length (SEM=0.116, Fig 2b); total number of

229 lateral roots (SEM=0.029); total lateral root, length/primary root length (SEM 0.013

230 Fig 2c); number of laterals/primary root length (SEM=0.003) and mean lateral root

231 length (SEM=0.025).

232

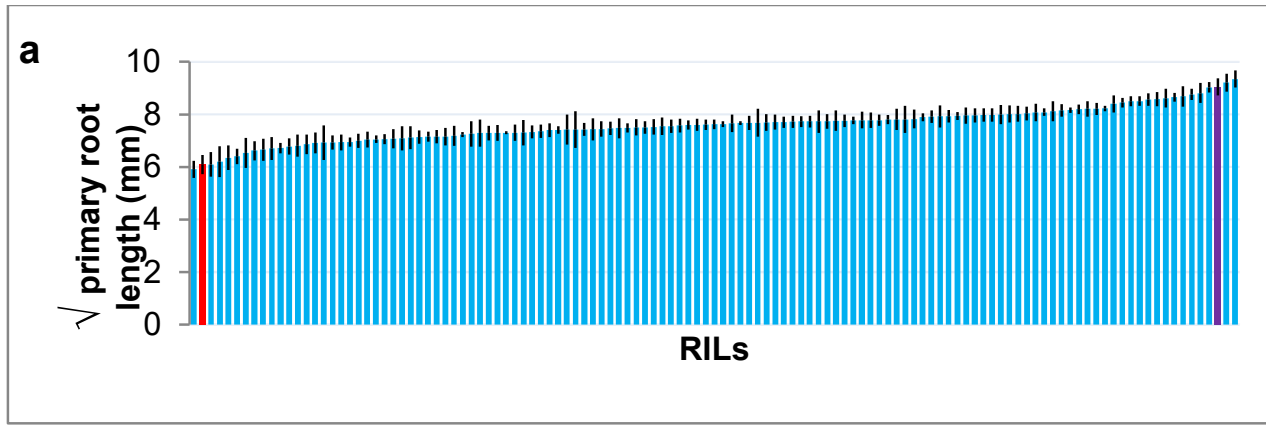

b
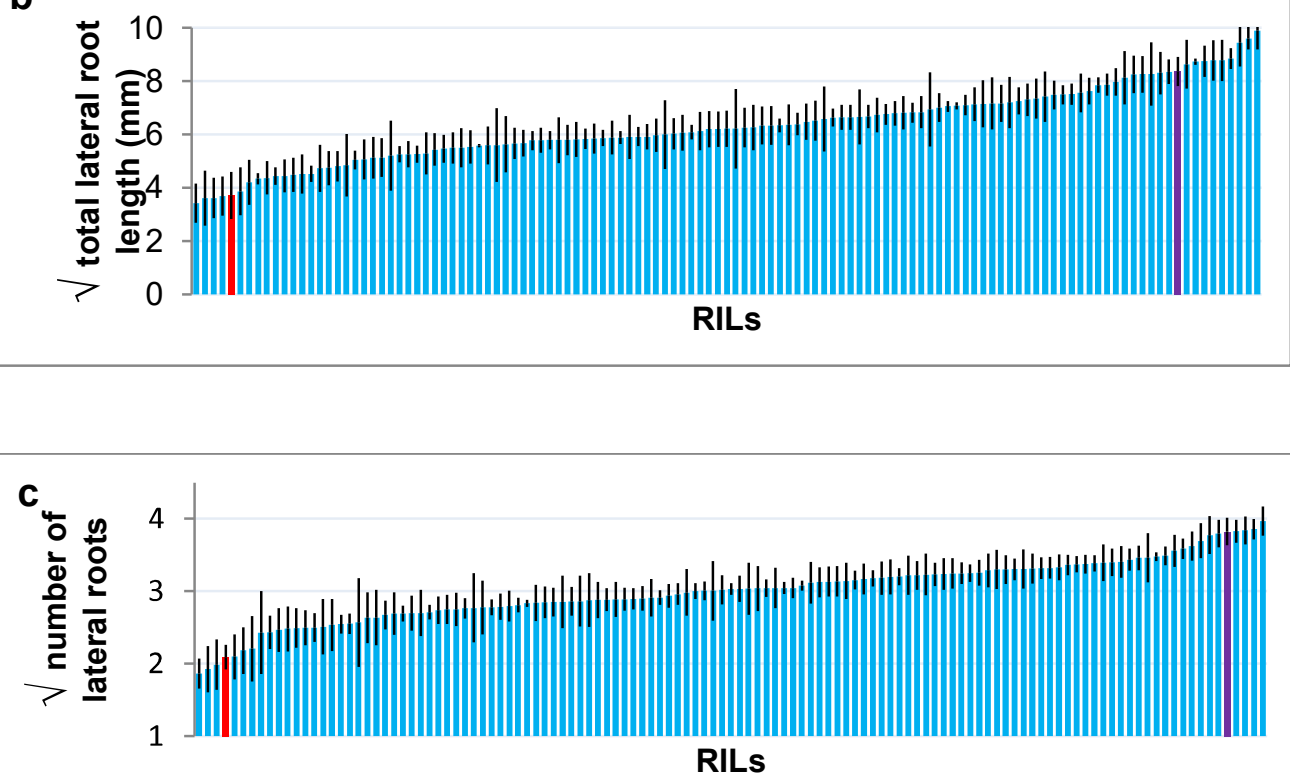
Fig. 2 Segregation of $14 \mathrm{~d}$ seedlings of the 125 RILs of the Saladin X roots. Red bars are the Saladin parent. Purple bars are the Iceberg parent and blue bars are the RILs; Error bars are SEM.

The chromosomal wide and genome wide significance at $\mathrm{P}<0.05$ was 0.994 .

This value when interpolated into the table by van Ooijen (1999) corresponding to the average chromosomal map length of $116 \mathrm{cM}$ gave a LOD score of $3.1 \mathrm{for}$ 
260 two QTLs explained $27.6 \%$ of the phenotypic variance for this trait. Three statistical

261 QTLs were found for LRND. These QTLs were on LG 7b, 8b and 9, explaining 33.4

$262 \%$ of the phenotypic variation for the trait and were labelled LRND-01, LRND-02 \&

263 LRND-03. The $\mathrm{H}^{2}$ for the ratio LRND was 0.24. For MLRL two statistical QTLs were

264 identified on LG 8 and 9b and these QTLs were labelled MLRL-01 and MLRL-02. A

265 total of $21.1 \%$ of the phenotypic variance of the MLRL trait can be explained by

266 these two QTLs and the $\mathrm{H}^{2}$ score for MLRL was 0.24 (Table 1; Fig. 3).

Table 1 Statistical QTLs $(P<0.05)$ for root traits and their genetic

positions in $14 \mathrm{~d}$ old seedlings of the Saladin $\mathrm{x}$ Iceberg mapping population.

\begin{tabular}{|c|c|c|c|c|c|c|}
\hline $\begin{array}{c}\text { QTL } \\
(P<0.05)\end{array}$ & $\begin{array}{l}\text { LOD } \\
\text { score }\end{array}$ & $\begin{array}{l}\text { Linkage } \\
\text { Group }\end{array}$ & Position (cM) & Associated markers & $\begin{array}{c}\text { Allelic } \\
\text { contribution }\end{array}$ & $\begin{array}{c}\% \\
\text { phenotype } \\
\text { explained }\end{array}$ \\
\hline PRL-01 & 5.82 & $7 b$ & $33.5-35.5$ & 7_LS1_750;39 & Iceberg & 17.1 \\
\hline PRL-02 & 3.84 & $9 a$ & $10.0-10.5$ & $\begin{array}{c}\text { AQYG-OP3 } \\
\text { 9_LS1_319;53 }\end{array}$ & Iceberg & 11.1 \\
\hline PRL-03 & 3.38 & $5 b$ & $0.0-1.0$ & $\begin{array}{l}\text { E35M47_191i } \\
\text { E45M60_160i }\end{array}$ & Saladin & 8.4 \\
\hline PRL-04 & 3.27 & $8 a$ & $27.4-24.4$ & $\begin{array}{c}\text { ARRK-OP4 } \\
\text { AKDB-OP4 } \\
\text { BVTF-OP4 } \\
\text { E35M61_280s }\end{array}$ & Iceberg & 7.8 \\
\hline PRL-05 & 3.26 & $4 b$ & $14.9-15.9$ & $\begin{array}{c}\text { 4_LS1_324;23 } \\
\text { AVZB-OP4 }\end{array}$ & Iceberg & 7.5 \\
\hline PRL-06 & 3.22 & $2 c$ & $35.3-35.3$ & 2_LS1_664;11 & Iceberg & 8.3 \\
\hline TLL-01 & 8.83 & $9 b$ & $12.8-12.8$ & 9_LS1_694;52 & Saladin & 23.8 \\
\hline TLL-02 & 3.24 & $5 b$ & $0.0-1.0$ & $\begin{array}{l}\text { E35M47_191i } \\
\text { E45M60_160i }\end{array}$ & Saladin & 7.2 \\
\hline TLL-03 & 3.2 & $3 a$ & $13.7-13.7$ & $\begin{array}{c}\text { AVSI-OP3 } \\
\text { 3_LS1_14;15 }\end{array}$ & Saladin & 7.6 \\
\hline LRLD-01 & 6.78 & $9 b$ & $8.6-12.8$ & 9_LS1_392;52 & Saladin & 19.0 \\
\hline
\end{tabular}




\begin{tabular}{|c|c|c|c|c|c|c|}
\hline LRLD-02 & 3.33 & $4 a$ & $5.1-5.1$ & BSCC-OP3-1 & Saladin & 8.6 \\
\hline LRND-01 & 4.13 & $8 b$ & $10.0-12.0$ & $\begin{array}{c}\text { E45M59_265i } \\
\text { AKQB-OP4 }\end{array}$ & Saladin & 10.4 \\
\hline LRND-02 & 4.02 & $7 b$ & $31.7-31.7$ & E35M47_244i & Iceberg & 8.7 \\
\hline LRND-03 & 3.97 & $9 a$ & $10.5-11.2$ & $\begin{array}{l}\text { 9_LS1_470;53 } 53 \text { 53 } \\
\text { 9_LS1_496 }\end{array}$ & Saladin & 14.3 \\
\hline MLRL-01 & 4.38 & $9 b$ & $7.4-7.4$ & BEMX-OP4 & Saladin & 10.8 \\
\hline MLRL-02 & 4.18 & $8 a$ & $1.0-3.1$ & $\begin{array}{c}\text { 8_LS1_591;48 } \\
\text { 8_LS1_58;49 } \\
\text { 8_LS1_229;49 }\end{array}$ & Saladin & 10.3 \\
\hline
\end{tabular}

269 Trait abbreviations are PRL (primary root length), TLL (total lateral root length),

270 LRLD (lateral root length density), LRND (lateral root number density) and MLRL

271 (mean lateral root length).

272 Four linkage groups contained regions with clustered QTLs, namely LG 5a,

$2737 b, 9 a$ and 9b (Table 1, Figure 3) highlighting regions of interest.

$5 b$

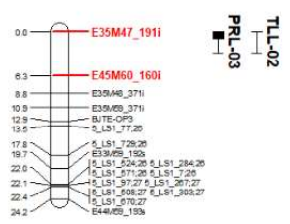

$7 b$

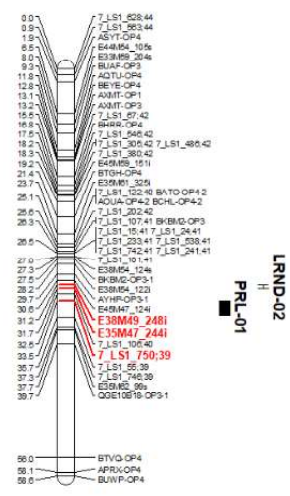

$9 a$

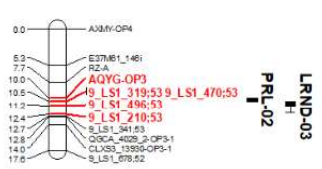

$9 b$

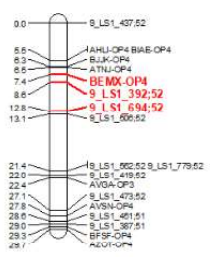

Fig 3. Clustered QTL positions and associated markers for the root

traits on the linkage map of the Saladin $x$ Iceberg mapping population. 
278 Statistical (black bars) QTL positions in centimorgans (cM) on the Saladin x

279 Iceberg linkage map. The solid blocks are the 1-LOD threshold (LOD score of

280 3.2), the outer intervals are the 2-LOD threshold. The markers in bold red are

281 those associated with the significant LOD of the QTLs. Abbreviations of traits

282 are PRL (primary root length), TLL (total lateral root length), TNL (total number

283 of lateral roots), LRLD (lateral root length density), LRND (lateral root number

284 density) and MLRL (mean lateral root length).

\section{Discussion}

The study identified 16 statistical QTLs associated with early stage rapid root 288 development in an intra-specific $L$. sativa mapping population. The markers associated with these traits could be used for marker assisted selection in breeding programmes in the future should an increase in root growth prove to be associated with better establishment in field grown lettuce transplants.

The study has identified genetic potential, within the intra-specific cross in a

2D assay that could be utilised within a breeding programme. Further studies would need to be undertaken however, to better understand what interaction the environment has on these traits in field conditions. The 2D high-throughput assay used in this study allows the rapid analysis of root traits in seedlings in a time, cost and labour efficient manner compared with other techniques, such as computed tomography (CT) 3D analysis (Mooney et al., 2012). Over 762 germinated seedlings were sown in $<6 \mathrm{~h}$, covering an area $<1.5 \mathrm{~m}^{2}$ at a cost of $<£ 0.50$ per seedling. This technique offers greater efficiency than sand or soil pot grown root analysis, which 
increases area use, labour and time costs dramatically as the roots need to be washed and separated before imaging/measuring can be accomplished.

In directly seeded crops the ability to produce a longer tap root early may be advantageous. Greater primary root length observed in $14 \mathrm{~d}$ old seedlings using the pouch system has been positively correlated with root emergence, faster establishment and increased seed yield in field grown Brassica napus (Thomas et al. 2016). Increased primary root length in seedlings potentially allows root access to deeper water resources (Johnson et al. 2000). Cultivated lettuce was described by Jackson (1995) as having a short tap root compared to its wild progenitor L. serriola. This study has observed significant difference within a $L$. sativa intra-specific cross for primary root length that may allow the ability to explore deeper soil layers and allow faster establishment and emergence in field grown lettuce. Six QTLs were identified for increased primary root length of which one was contributed by the Saladin parent on LG 5b (PRL-03) while the others were contributed by the Iceberg parent and were located on LG 7b (PRL-01), 9 (PRL-02), 8 (PRL-04), 4b (PRL-05) and 2c (PRL-06). Further work would be needed to identify if the RIL lines with a greater primary root length trait in $14 \mathrm{~d}$ seedlings emerge and establish faster and develop a longer, deeper tap root at maturity in the field.

In transplanted lettuce where mechanical pruning of the primary root often occurs (Kerbiriou et al. 2013), recovery of the root:shoot ratio may be governed by the plants ability to rapidly replace lost root mass through lateral root growth. Establishment is also dependent on the crops ability to regenerate lateral roots during establishment (Orzolek 1991) allowing early capture of the resources available to further optimise shoot growth. Longer total root length of wheat seedlings in a growth pouch assay has been associated with increased yield and shoot 
biomass in the field (Xie et al. 2017). Five statistical QTLs were found that were linked with total lateral root length. Two QTLs were located along LG 9b (TLL-01 and LRLD-01) overlapping the same region and probably represent a single locus. The further three QTLs were located on LG 5b (TLL-02), 3 (TLL-03) and 4 (LRLD-02).

Decapitation of the root tip from primary lateral roots in lettuce seedlings has been shown to slow and even cease the emergence of any further secondary or tertiary lateral roots along the length of the decapitated root (Biddington \& Dearman 1984). The pruning of the lateral roots often occurs as a consequence of the mechanical separation of adjacent peat blocks in the process of transplanting lettuce. Hence, breeding for cultivars that can regenerate greater numbers of primary lateral roots more efficiently may be a desirable trait that helps plants establish more rapidly. There were three individual statistical QTLs linked to the total number of lateral roots. The QTLs were for the lateral root number density trait and were located on LG 8b, (LRND-01), 7b (LRND-02) and 9 (LRND-03). LRND-01 and LRND-03 were contributed by the Saladin parent, while LRND-02 was contributed by the Iceberg parent.

The ability of a lettuce transplant to produce fewer longer lateral roots (greater MLRL) may be advantageous. Fitter et al. (1991) suggested exploitation efficiency (amount of soil exploited per carbon unit cost of root) may be beneficial to crops. If lettuce transplants were able to produce fewer longer lateral roots with less branching following transplanting, then the plant would be able to utilise the resources captured mainly on shoot growth. There were two statistical QTLs identified for MLRL in this study that may be beneficial to exploitation efficiency in lettuce transplants. The first was located on LG 9b (MLRL-01) and the second was on LG 8 (MLRL-02). 
The overlying region on LG $9 \mathrm{~b}$ between 8.6 and $12.8 \mathrm{cM}$ for both the total

lateral root length and lateral root length density traits (3 QTLs), but not total number of lateral roots, lateral root number density and mean lateral root length suggests that this region is genetically involved with increased individual lateral root length or decreased branching/topology, which would indicate this region could be exploited to increase the root exploration potential (Fitter \& Stickland 1991) in lettuce transplants.

Only one of the six statistical QTLs identified in this paper for primary root length (i.e. tap root length) was located on LG 2-(LG 2c), where QTLs for the trait were identified by Johnson et al. (2000), however, the study cannot identify if the loci are the same. One of the QTL in this study located to the region towards the end of linkage group 2c (35.3 cM) close to the area on LG 2 where Johnson et al. (2000) had mapped a QTL associated with tap root length contributed by the wild parent. A further QTL identified in this study (TLL-03) mapped to LG 3 (13.7 cM), which is in proximity to the QTL identified by Johnson et al. (2000) associated with number of lateral roots.

The two QTLs identified on the LG 5 group (5b), PRL-03 and TLL-02 locate to the same LG as a QTL linked to lateral root length and lateral root number observed by Wei et al. (2014). Johnson et al. (2000) also located a QTL on LG 5 that was linked to lateral root number in the lower soil profile contributed by the wild relative $L$. serriola. This region is therefore strongly linked to lateral root emergence and growth in both cultivated and wild parents. The QTLs PRL-02, TLL-01, LRLD-01, LRND-03 and MLRL-01 located on the same LG (LG 9 and 9b) to the QTLs identified by Wei et al. (2014) linked to general root growth. Our study identifies this region as being linked with all the root growth traits - primary root growth, lateral root growth and lateral root emergence. 
377 respectively for the three measured traits primary root length, total lateral root length

378 and total number of lateral roots indicating that these lines would be the best

379 candidates to use in a gene expression study to identify the genes underlying the

380 QTLs and others that are involved with increased root growth rate traits. Increased

381 root growth traits could reduce the period of the recovery phase, caused by

382 transplant shock (van lersel 1998), by quickly restoring the root:shoot ratio and

383 therefore increasing crop uniformity by reducing transplant establishment time.

384 However; certain negative possibilities could occur. In a rapid rooting line, the

385 increase in growth could mean more lateral roots are pruned leading to an enhanced

386 transplant shock, meaning no benefit to establishment would apply. These concerns

387 would need further studies to be resolved.

\section{Conclusion}

A rapid rooting phenotype may be beneficial to the establishment of lettuce transplants in commercial field production. Such a phenotype could reduce transplant shock and alleviate reduction in shoot growth due to mild abiotic stresses that occur in the field. The use of a high throughput rooting growth pouch assay revealed significant genetic variation in a Saladin X Iceberg cross RIL population to identify QTLs linked to the traits associated with a rapid rooting phenotype in $14 \mathrm{~d}$ old seedlings. A total of 16 statistical QTLs were identified. The statistical QTLs were located across 9 different LGs representing loci on 7 of the 9 L. sativa chromosomes.

398 DNA markers linked to these rooting traits were identified, which could allow 399 breeders to select for a rapid establishment phenotype. 

programmes and may be of more direct utility compared to markers from inter-

402 specific crosses, which contains genetic material from the wild L. serriola parent 403 (Atkinson et al. 2013).

\section{Conflicts of interest}

$405 \quad$ The authors are not aware of any conflict of interest in this study via funding 406 or any affiliations.

\section{References}

409 ImageJ. Biophotomics International 11(7):36-42. Atkinson.J.A, Wingen.L.U, Griffiths.M, Pound.M.P, Gaju.O, Foulkes.M.J, Le 412 Gouis.J, Griffiths.S, Bennett.M.J, King.J \& Wells.D.M (2015) Phenotyping pipeline 413 reveals major seedling root growth QTL in hexaploid wheat. Journal of Experimental 414 Botany 66:2283-2292.

415 Atkinson.L.D, McHale.L.K, Truco.M.J, Hilton.H.W, Lynn.J, schut.J.W, 417 Michelmore.R.W, Hand.P \& Pink.D.A.C (2013) An intra-specific linkage map of 418 lettuce (Lactuca sativa) and genetic analysis of postharvest discolouration traits. 419 Theor. Appl. Genet. 126(11):2737-2752. 
422 seedlings following root pruning. Annals of Botany 53:663-668.

423

Fitter.A.H, Stickland.T.R, Harvey.M.L \& Wilson.G.W (1991) Architectural

425

analysis of plant root systems 1 . Architectural correlates of exploitation efficiency.

426

New Phytologist 118:375-382.

427

Fitter.A.H \& Stickland.T.R (1991) Architectural analysis of plant root systems

429

430

2. Influence of nutrient supply on architecture in contrasting plant species. New phytologist 118:383-389.

431 establishment. Sci. Agric. (Piracicaba, Braz.) 55:116-120.

434

Jackson (1995) Root architecture in cultivated and wild lettuce (Lactuca spp.).

436

Plant, Cell and Environment 18(8):885-894.

437

Jansen.R.C \& Stam.P (1994) High resolution of quantitative traits into

439 multiple loci via interval mapping. Genetics 136:1447-1455. 
Johnson.W.C, Jackson.L.E, Ochoa.O, van Wijk.R, Peleman.J, St.Clair.D.A, \&

442 Michelmore.R.W (2000) Lettuce, a shallow-rooted crop, and Lactuca serriola, its wild

443 progenitor, differ at QTL determining root architecture and deep soil water

444 exploitation. Theor. Appl. Genet. 101:1066-1073.

445

446

Kerbiriou.P.J, Stomph.T.J, Lammerts van Bueren.E.T \& Struik.P.C (2013)

447 Influence of transplant size on the above- and below-ground performance of four

448 contrasting field-grown lettuce cultivars. Frontiers in Plant Science 4(379):1-16.

449

Le Marié.C, Kirchgessner.N, Marschall.D, Walter.A \& Hund.A (2014)

451 Rhizoslides: paper-based growth system for non-destructive, high throughput

phenotyping of root development by means of image analysis. Plant Methods

453 10(13):1-16.

454 computed tomography to non-invasivley image 3-D root systems architechture in soil. Plant Soil 352:1-22. 
464 8(4):495-498.

465

$467 \quad 1(1): 78-81$.

Schneider.C.A, Rasband.W.S \& Eliceiri.K.W (2012) NIH image to ImageJ: 25 years of image analysis. Nature Methods 9(7):676-682. transplant shock in tomato seedlings. Journal of Vegetable Science 11(3):41-56. assisted selection to introgress rice QTLs controlling root traits into an Indian upland

477 rice variety. Theor. Appl. Genet. 112:208-221.

480 Virk.D.S (2013) QTLs associated with root traits increase yield in upland rice when 481 transferred through marker-assisted selection. Theor. Appl. Genet. 126(1):101-108. 
484 Nightingale.M, Dupuy.L.X, Hammond.J.P, White.P.J \& Broadley.M.R (2016) High-

485 throughput phenotyping(HTP) identifies seedling root traits linked to variation in seed

486 yield and nutrient capture in field-grown oilseed rape (Brassica napus L.). Annals of 487 Botany 118:655-665.

488

Van lersel.M (1998) Auxins affect posttransplant shoot and root growth of

490 Vinca seedlings. HortScience 33(7):1210-1214.

Van Ooijen.J.W (1999) LOD significance thresholds for QTL analysis in

493

experimental populations of diploid species. Heredity 83:613-624.

494

495

Wei.Z, Julkowska.M.M, Laloë.J-O, Hartman.Y, de Boer.G-J, Michelmore.R.W, 496

van Tienderen.P.H, Testerink.C \& Schranz.M.E (2014) A mixed-model QTL analysis

497 for salt tolerance in seedlings of crop-wild hybrids of lettuce. Molecular Breeding $498 \quad 34(3): 1389-1400$

499

500

Zeng.Z-B (1994) Precision mapping of quantitative trait loci. Genetics

501 136:1457-1468.

502 
504 approaches to phenotyping root system architecture. Current Opinion in Plant

505 Biology 14:310-317. 